\title{
EXPERIMENTAL STUDIES OF BEAM-BEAM EFFECTS IN THE TEVATRON
}

\author{
Xiaolong Zhang, Tanaji Sen,Vladimir Shiltsev, Meiqin Xiao,Yuri Alexahin, FNAL, Batavia, IL 60510, USA \\ Frank Schmidt, Frank Zimmermann, CERN, Geneva, Switzerland
}

\begin{abstract}
The long-range beam-beam interactions limit the achievable luminosity in the Tevatron. During the past year several studies were performed on ways of removing the limitations at all stages of the operational cycle. We report here on some of these studies, including the effects of changing the helical orbits at injection and collision, tune and chromaticity scans and coupling due to the beam-beam interactions.
\end{abstract}

\section{GENERAL OBSERVATIONS}

The Tevatron is currently colliding 36 proton against 36 antiproton bunches, where either beam consists of 3 equally spaced trains of 12 bunches in a common single vacuum chamber. The two beams are separated by a helical orbit except at the two locations of High Energy Physics (HEP) experiments, where they collide head on. The beam-beam effect gains more and more importance as the Tevatron beam intensity continues to increase in the quest for higher luminosity [1]. Recently, the total beam intensities injected into the Tevatron has been slightly over $10 \times 10^{12}$ protons and $1.2 \times 10^{12}$ antiprotons, which, subsequently, yielded a peak luminosity of $43 \times 10^{30} \mathrm{~cm}^{-2} \mathrm{~s}^{-1}$ with over $8 \times 10^{12}$ protons and $0.9 \times 10^{12}$ antiprotons in collision. Intensive studies were carried out in recent months to understand and quantify limitations from beambeam effects.

\section{Beam Loss Through Shot}

The typical beam loss through the shot setup is shown below in Figure 1. The red curve is the energy of the Tevatron, the green curve is the proton total bunch intensity, and the black is the total antiproton bunch intensity. The poor proton lifetime is caused by a limited dynamic aperture.

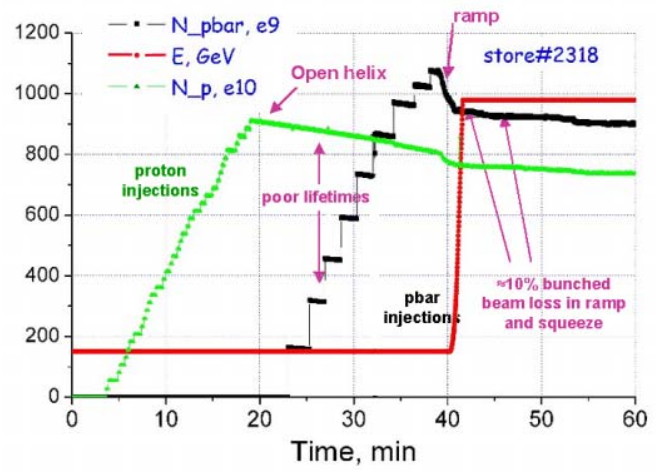

Figure 1: Beam intensity and loss during injection, ramping and squeeze
During ramping, there are about 10 12\% antiproton loss and about 5 7\% proton loss. There also is about 5\% combined beam loss during low beta squeeze and halo removing process.

The losses are smaller for shorter bunches $(\sim 30 \%$ of longitudinal emittance reduction will reduce losses to $\sim 3$ $4 \%$ ) and for smaller transverse emittance (almost no losses occur if the transverse emittances are less than 12 pi $\mathrm{mm}$ mrad, while typical emittances are $20-25$ pi $\mathrm{mm}$ mrad). Antiproton losses are much higher in case of insufficient separation.

\section{Antiproton Only}

A study with antiprotons only proved that the antiproton loss on the ramping is caused by the beambeam effect. Figure 2 shows the antipron intensity during ramping and squeezing. No bunched beam loss (red curve) is observed; some DC beam (black curve) is lost during ramping.

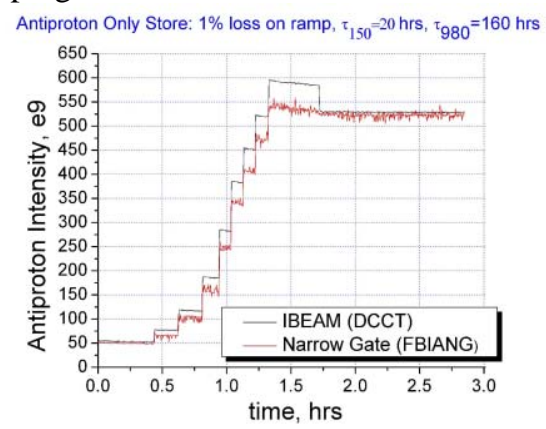

Figure 2: Beam intensity during antiproton only store.

Comparing the antiproton losses in Figures 1 and 2, we conclude that the loss is mainly caused by the presence of strong proton bunches.

\section{Inproving Sequence \#13}

The antiproton loss due to insufficient separation was also a problem at the initial stage of Tevatron Run II operation as shown in Figure 3.
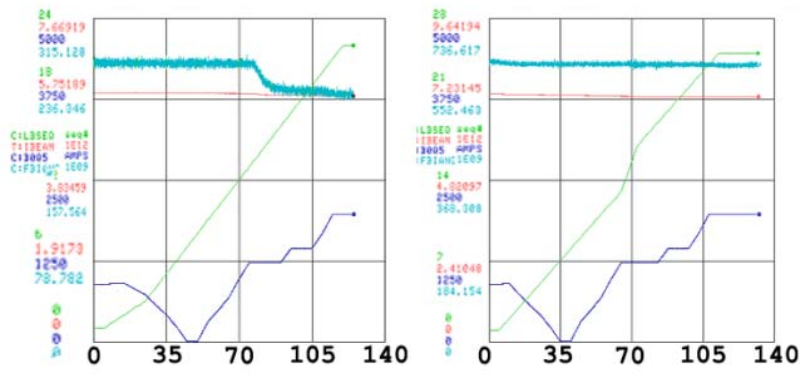

Figure 3: The antiproton losses due to the insufficient beam separation (left) and its solution (right). 
There was a huge antiproton loss during the ramp when the Tevatron energy was greater than $500 \mathrm{GeV}$ in ramping sequence \#13, which is illustrated by the cyan curve (C:FBIANG) on the left of Figure 3. After painstaking studies, the problem was identified as an insufficient beam separation. The solution was one more ramping break point with increased beam separation, which was added between ramping sequences \#13 and \#14. After this improvement, the loss due to beam-beam effects was eliminated as shown in the similar graph on the right of Figure 3.

\section{TUNE MEASUREMENT}

There are basically 3 systems for tune measurement in the Tevatron [2]. These include two Schottky systems, an old one at $21 \mathrm{MHz}$ and a new one at $1.75 \mathrm{GHz}$, and the tune meter which measures the beam betatron oscillation frequency by FFT and is able to measure the tune bunch by bunch. The beam can be excited using either a stripline kicker or the Tevatron electron lens [3].

\section{Tune Scan}

One can find the best working point for protons and antiprotons by a tune scan. Figure 4 is the result of one of the tune scans carried out at the end of a HEP store.

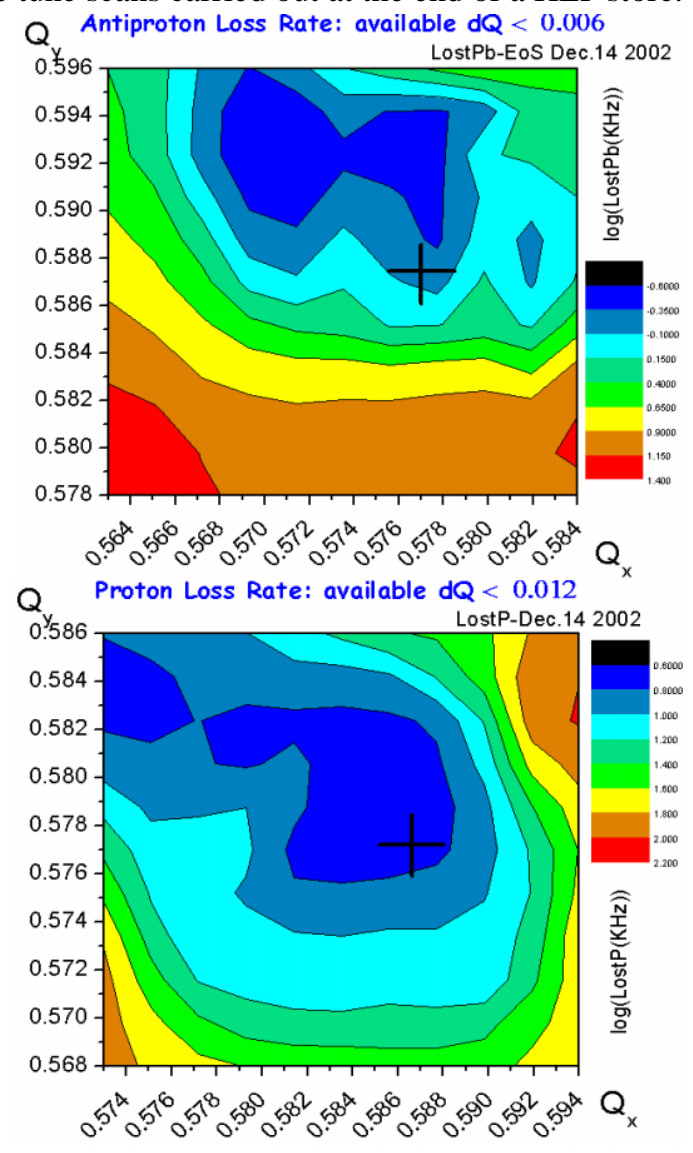

The tune scan shown in the figure above displayed the loss versus the working point. The proton and antiproton losses were measured by the CDF detector. The top figure shows the loss rate of protons and the bottom one that of antiprotons. The blue color in the above graph indicates small losses and the crosses stand for the initial nominal working point at the time of the tune scan study. It indicates that there is some room for daily operational optimization of the working point.

\section{Tune Diagram}

By exciting or gating individual bunches, we can measure the bunch by bunch tune of the $36 \times 36$ bunches. The graph below shows the tunes of the 36 antiproton bunches at the end of the store.

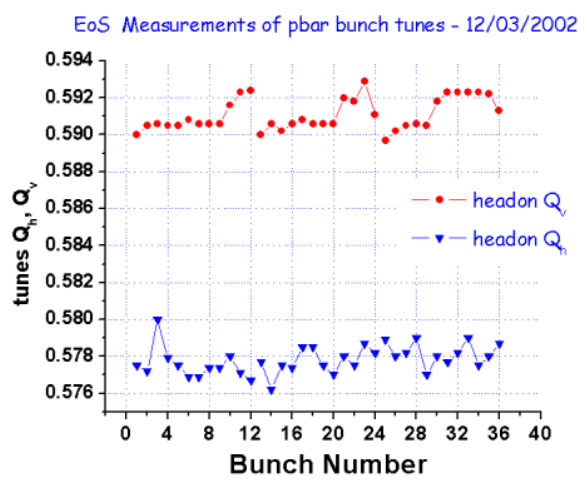

Figure 5: The bunch by bunch tune of the antiproton bunches

For this study, we 'tickled' each antiproton bunch and measured the tune spectrum by the Schottky signal monitor. We found that the vertical tune of the first bunch in the train of 12 bunches was lower and a few bunches at the end of the train had higher tune. The magnitudes of the the tune shifts and the fact that leading and trailing bunches are strongly affected agree with the simulations[1][4]. The first bunch had a lower tune because it missed one long range beam-beam collision and the higher tune was due to the fact the last few bunches had smaller emittances.

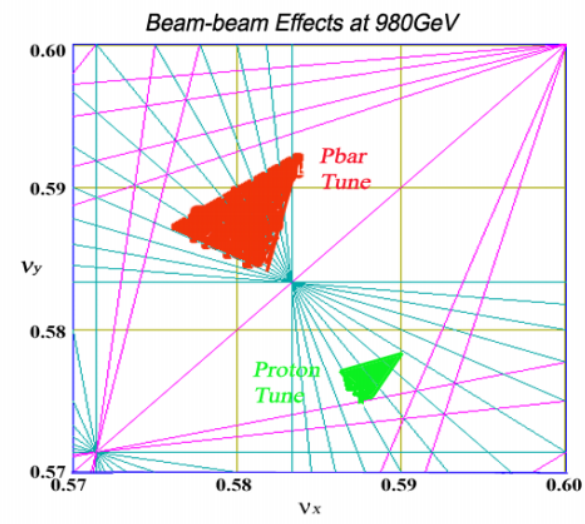

Figure 6: The tune diagram of the proton and antiproton bunches

The Figure 6 shows the usual working points of the Tevatron for proton and antiproton bunches. The tunes of both beams overlap $12^{\text {th }}$ order resonance lines. 


\section{Emittance Scallops}

Another beam-beam effect observed in the Tevatron is that occasionally antiproton emittances blow up when the tunes of the antiproton bunches are not optimized. The emittance blows up slowly, over about 5 15min. Afterwards we observe an enhanced emittance variation from bunch to bunch, which is shown in Figure 7, and is called the emittance scallop effect.

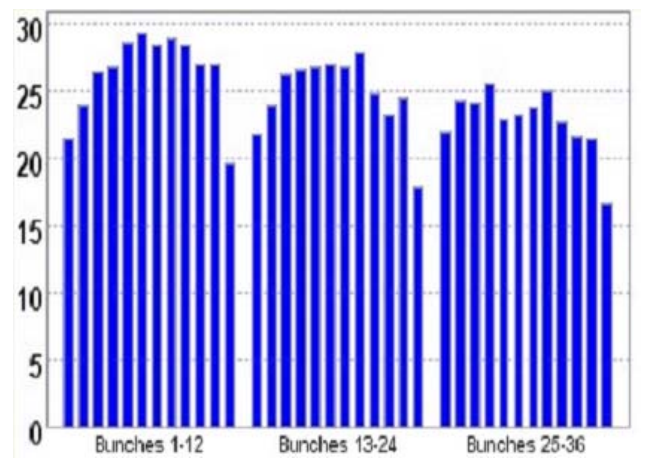

Figure 7: Emittance scallops of Antiproton bunches measured by Tevatron Fly wire.

The emittance blow up degrades the luminosity. Thus, some studies were carried out trying to use the TEL as beam-beam compensation to eliminate these emittance blow ups [5]. Moreover, we must be careful in choosing the Tevatron working point. Unfortunately, the tunes and coupling, etc. are drifting in time [6]. It takes a lot of effort to re-optimize the Tevatron in every other week.

\section{EFFECTS OF HELIX SIZE}

One of the most direct studies on long range beambeam effect was carried out by investigating the effects of the helix size on the colliding beams. Figure 8 below shows the proton and antiproton losses versus the helix sizes of the Tevatron beam orbit for $980 \mathrm{GeV}$ at the end of HEP store.

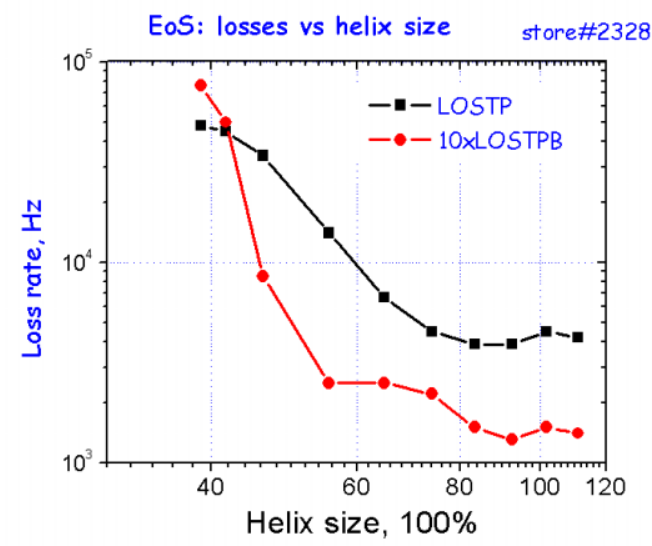

Figure 8: The beam losses versus the beam separation.

The loss rates are low and approximately flat within $82 \sim 110 \%$ of the nominal helix size, and they went up as the helix size is decreased, which agrees with theoretical expectations, since much stronger long range beam-beam effects cause larger beam-beam tune shifts and shift the more of the beam onto nonlinear resonances, as the beam separation gets smaller.

\section{EFFECTS OF THE CHROMATICIY}

The chromaticity is the main factor which causes proton beam losses during injection at $150 \mathrm{GeV}$ when the proton

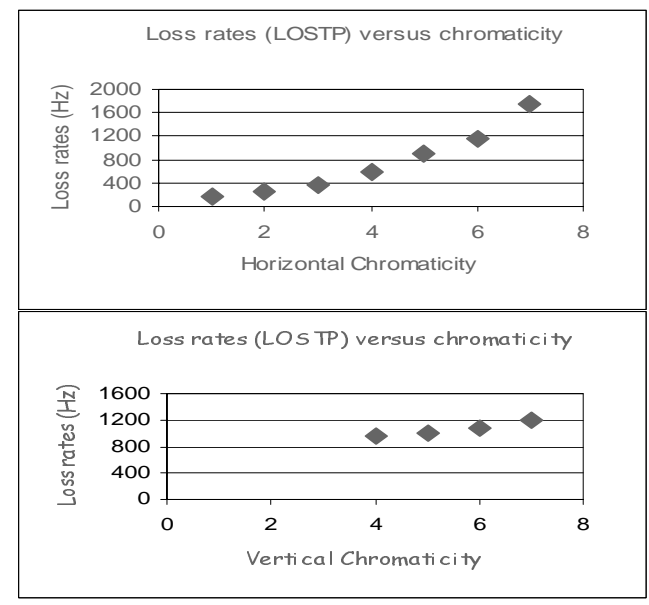

beam is on the helical orbit. Generally, the lower the chromaticity, the less is the proton loss. The Figure 9 above shows a chromaticity scan at injection energy on the proton orbit for coalesced bunches. One can see that when the horizontal chromaticity was lowered to 1 unit, we had minimum losses. However, we could lower the vertical chromaticity onuly down to 4 units, where the head-tail instability occurred. For normal Tevatron operation, we now apply transverse beam feedbacks, in order to be able to lower the chromaticity to improve the proton lifetime at injection.

\section{SUMMARY}

Beam-beam effects are the key to further Tevatron Run II upgrades. Intensive studies were carried out on beam separation scheme, working points, beam emittance control, etc. We have plans and a schedule for further beam-beam studies aimed at improving the Tevatron performance with increasing beam intensity, so as to provide a higher luminosity and stable operation.

\section{REFERENCES}

[1] Tanaji Sen et al., this conference, TPPB069 and TPPB067.

[2] Cheng-Yang Tan, this conference, WPPG052

[3] X. Zhang et al., this conference, TPPB076

[4] Alexahin Yuri, FERMILAB-Pub-00/120-T(2000)

[5] K. Bishofberger et al., this conference, TPPB083 and MOPA011

[6] Mike Martens, this conference, FPAB031 